\title{
Do Inner Ear and Mastoid Bone Structural Variations Have a Determining Role on Vestibular Symptoms of Migraine?
}

\author{
(1) Zehra Cinar, (1) Cigdem Kalaycik Ertugay, (1) Ozgür Yigit, (1) Yesim Karagoz*, \\ (1) Osman Ozgur Yalin**
}

University of Health Sciences Turkey, Istanbul Training and Research Hospital, Clinic of Otorhinolaryngology Head and Neck Surgery, Istanbul, Turkey

*University of Health Sciences Turkey, Istanbul Training and Research Hospital, Clinic of Radiology, Istanbul, Turkey

** University of Health Sciences Turkey, Istanbul Training and Research Hospital, Clinic of Neurology, Istanbul, Turkey

\section{Abstract}

\begin{abstract}
Aim: To investigate the possible relationship between morphological changes caused by anatomical structural differences and functional impairment in Vestibular migraine (VM) and Chronic Migraine (CM) disease.

Methods: A total of 44 patients (VM group; 23 and CM group; 21) included in the study. The medical records of patients with CM and VM between 2016-2018 were retrieved from the digital database of the otorhinolaryngology and neurology clinics. The mastoid bone and inner ear measurements in temporal bone high resolution computed tomography and inner ear magnetic resonance imaging were performed in our study. The two groups were compared radiologically.
\end{abstract}

Results: Right cochlear height in the VM group was significantly higher than the CM group. The left vestibular height in the VM group was significantly higher than the CM group.

Conclusion: The reason why not all patients with CM have vestibular complaints may be due to some differences in the inner ear anatomical structure.

Keywords: Vertigo, migraine disorders, cochlea, vestibule, labyrinth, ear, inner, mastoid

\section{Introduction}

Migraine is a specific neurological disease and diagnosis is made according to the widely accepted International Classification of Headache Disorders 3rd Edition (ICHD-3). If the attack frequency is more than 15 days per month for more than three months, it is diagnosed as chronic migraine $(C M)(1,2)$. Vestibular migraine $(V M)$ is one of the most common causes of episodic vertigo in adults and children (3). The diagnostic criteria for VM were proposed by Neuhauser in 2001 and revised by the Bárány NeuroOtology Society in 2012, and diagnostic criteria were established in 2013 with the International Headache Society. Accordingly, the diagnostic criteria combine the typical signs and symptoms of migraine with vestibular symptoms lasting 5 minutes to 72 hours and exclusion of other causes of headache (4). Vestibular migraine is considered as the second most common cause of vertigo (5).

Based on the idea of possible similar pathophysiology, the literature focused on the differential diagnosis of Meniere's Disease and VM (6-8). On the other hand, there are few studies comparing $\mathrm{CM}$ and VM radiologically and clinically. Carvalho et al. (9) investigated the relationship between vestibular symptoms and migraine subtypes, and detected a greater frequency of vestibular symptoms in CM patients. However, we do not know the reason for not having vestibular complaints in all migraine patients. Some reports showed radiological increased activity

Yazışma Adresi/Address for Correspondence: Zehra Cinar, University of Health Sciences Turkey, Istanbul Training and Research Hospital, Clinic of Otorhinolaryngology Head and Neck Surgery, Istanbul, Turkey 
involvement in some regions of the brain in patients with VM $(10,11)$. Based on this information, we hypothesized that the reason might be several anatomical differences. Nevertheless, there is a lack of literature about this issue.

Based on these facts, the mastoid bone and inner ear measurements in temporal bone high resolution computed tomography (HRCT) and inner ear magnetic resonance imaging (MRI), as well as measurements of the internal acoustic canal (IAC) and cranial nerves in it were performed in our study. Both groups were compared radiologically. This study aims to reveal the possible relationship between morphological changes caused by anatomical structural differences and functional impairment in these two diseases.

\section{Main points}

$\mathrm{CM}$ is a frequent neurological disease. $\mathrm{VM}$ has the typical signs and symptoms of CM combined with vestibular symptoms. In the current study right cochlear height in the VM group was significantly higher than the $C M$ group $(p<0.05)$. The left vestibular height in the VM group was significantly higher than the CM group $(p<0.05)$. The reason for not having vestibular complaints in all CM patients might be several anatomical differences. This is the first study that investigated the inner ear and mastoid bone anatomical variations between VM and CM.

\section{Methods}

For this study, the approval was obtained from the local ethics committee of a tertiary hospital on 09.03.2018 (Decision number: 1198). This study was a retrospective comparative study conducted using the medical records of patients diagnosed with VM and CM disease. So informed consent was not taken.

\section{Study Design}

This research is a retrospective comparative study conducted by accessing the medical records of patients with CM diagnosis who were followed up and treated in the neurology clinic and the patients diagnosed with VM who were treated and followed up in the tertiary hospital Otorhinolaryngology-Head and Neck Surgery Clinic and Neurology Clinic between 2016-2018. The medical records of patients were retrieved from the digital database of the otorhinolaryngology and neurology clinics.

\section{Patient Selection}

The patients were evaluated by neurologists and headache specialists from the tertiary hospital Neurology Clinic and Vertigo Clinic of Otorhinolaryngology-Head and Neck Surgery Clinic and diagnosed with VM and CM according to ICHD-3 diagnostic criteria (1).

A neurology specialist who worked in the headache outpatient clinic made full neurological examinations, and differential diagnosis of $\mathrm{CM}$ and VM. An otolaryngologist evaluated these patients in the vertigo outpatient clinic and performed detailed otomicroscopic and vestibular examinations by using videonystagmography. The differential diagnosis of Meniere's disease, benign paroxysmal positional vertigo and other possible etiologies and related conditions of vertigo were made by the consensus of otolaryngologist, neurology specialist and radiologist.

Patients who had an ongoing infection, were pregnant, had autoimmune disorders, history of the previous otologic and cranial surgery, Meniere's disease, benign paroxysmal positional vertigo-like dizziness pathology, chronic neurophysiological disease, psychiatric pathologyrelated headache, patients who were $<18$ and $>65$ years old were excluded.

All patients were examined in terms of clinical characteristics such as disease duration, headache characteristics, pain localization, attack frequency, use of analgesics, and treatment options by a neurologist who worked in the headache clinic. Pain severity was evaluated with the visual analog scale. Also, the migraine disability assessment scale and the beck depression scale were filled out by the patients, and allodynia was evaluated using the Turkish version of the validated 12-item allodynia symptom checklist (12).

Migraine, VM and CM diagnoses were made by an experienced neurologist based on ICHD-3 diagnostic criteria (1). Patients with secondary headaches and other primary headache disorders were excluded. Other vestibular causes that could accompany VM were excluded by otolaryngological examination and audio-vestibular tests performed by the relevant specialists.

Computed Tomography Parameters and Data Analysis

Measurements of the labyrinth performed with temporal bone HRCT sections were determined using the methods described in the literature on the raw images and the constructed images created by the relevant researcher at the workstation (13-16). These measurements were chosen because previous studies have demonstrated their utility in identifying detailed inner ear abnormalities (1419).

Temporal bone HRCT examinations were performed on the patients with a multi-detector CT unit (Philips Ingenuity 128 CT scanner, Philips Healthcare, Cleveland, $\mathrm{OH}$ ) in the radiology clinic of our hospital. The tube voltage and current were $140 \mathrm{kV}$ and $124 \mathrm{mAs}$; field-of-view was in the range of $22-24 \mathrm{~cm}$. While the patient was in the supine position, axial sections with a slice thickness of $0.625 \mathrm{~mm}$ parallel to the orbitomeatal line were taken with $0.33 \mathrm{~mm}$ increments without overlap in the bone algorithm. At the workstation (GE Healthcare advantage workstation 4.3, 
Milwaukee, WI), raw data were reconstructed, and the radiologist performed inner ear and IAC measurements. CT measurements performed in this study were: Cochlear width and height, vestibular length and height, IAC proximal, mid and distal heights, $\mid \mathrm{ACl}$ ength cochlear apertura diameter, vestibular apertura diameter, distance between the tegmen-mastoid tip, the distance between sigmoid sinus anterior wall - external acoustic meatus, the distance between petrous apex - mastoid aditus medial wall. (Figure 1)

\section{Magnetic Resonance Imaging Parameters and Data analysis}

Due to the long and not always planar course extending from the brainstem to the cerebellopontine angle and from there to the fundus of the $I A C$, reconstruction of the nerve diameters and cross-sectional areas at varying levels is required in the evaluation of cranial nerves (20).

3D T2A-weighted raw images for temporal bone and cerebellopontine angle (SPA) taken with the routine protocol (TR: TE: FA: 0,7 mm slice thickness, $256 \times 128$ matrix) in MRI unit (1.5 T GE Healthcare Signa Hdxt, Milwaukee, $\mathrm{WI}$ ) were transferred to the workstation (GE Healthcare advantage workstation 4.3, Milwaukee, WI). In the reconstructed and sagittal-oblique angled projections, nerve traces were visualized in sections perpendicular to the neural structures within the IAC. Ellipsoid or circular

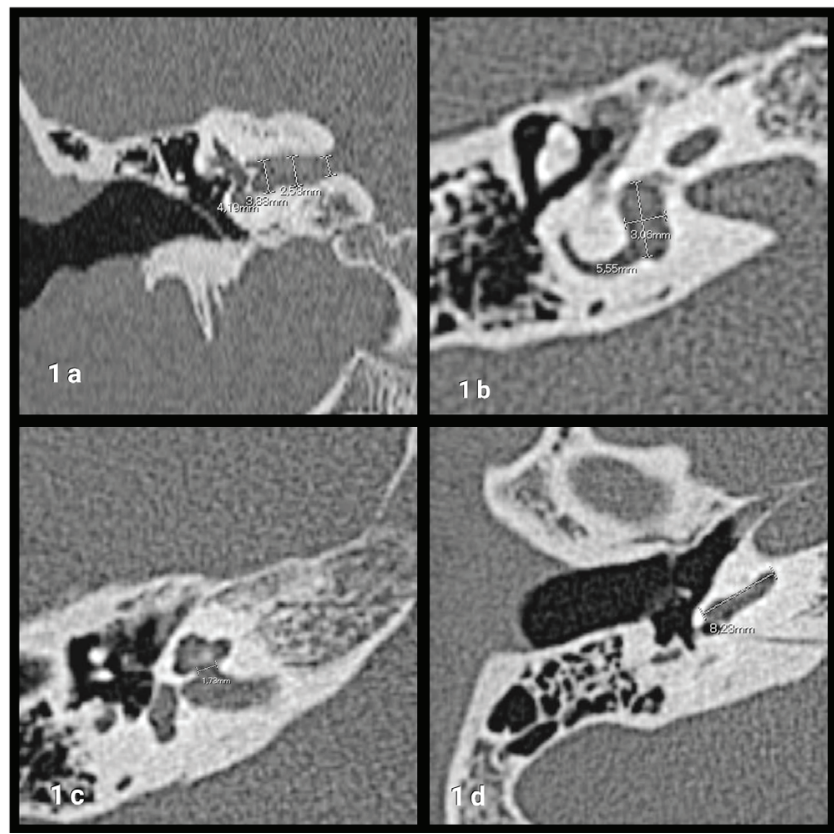

Figure 1. a. Proximal, middle and distal internal acustic canal height measurements in coronal multiplanar reconstruction image on temporal bone HRCT. b. Vestibule measurements in the axial section on HRCT. c. Cochlear nerve bone canal measurement on HRCT. d. Cochlear width measurement on HRCT $H R C T$ : High resolution computed tomography shaped surface areas of the nerve structures from the SPA system were tried to be measured from the same levels bilaterally. MRI measurements performed in this study were: Vestibulocochlear complex cross-sectional surface area (CSA), facial nerve CSA (cerebellopontin angle), superior and inferior vestibular nerves CSA's, IACcochlear nerve CSA (Figure 2).

\section{Statistical Analysis}

Statistical analyzes were made with the SPSS version 26.0 program. Compliance of quantitative variables to normal distribution was examined by histogram graphics and the Kolmogorov-Smirnov test. Mean, standard deviation, and median values were used while presenting descriptive analyzes of age, CT and MRI results. When comparing gender ratios between CM and VM groups, the Chi-square test was used. Age, CT and MRI results were compared between CM and VM groups using the MannWhitney $U$ test. The situations where the p-value was less than 0.05 were evaluated as statistically significant results.

\section{Results}

Twenty-three patients (15 women, 8 man; mean age $40.7 \pm$ standard deviation 12.8 , a total of 46 ears) diagnosed with $\mathrm{VM}$ and 21 patients diagnosed with CM headache (18 women, 3 men, mean age $40.7 \pm$

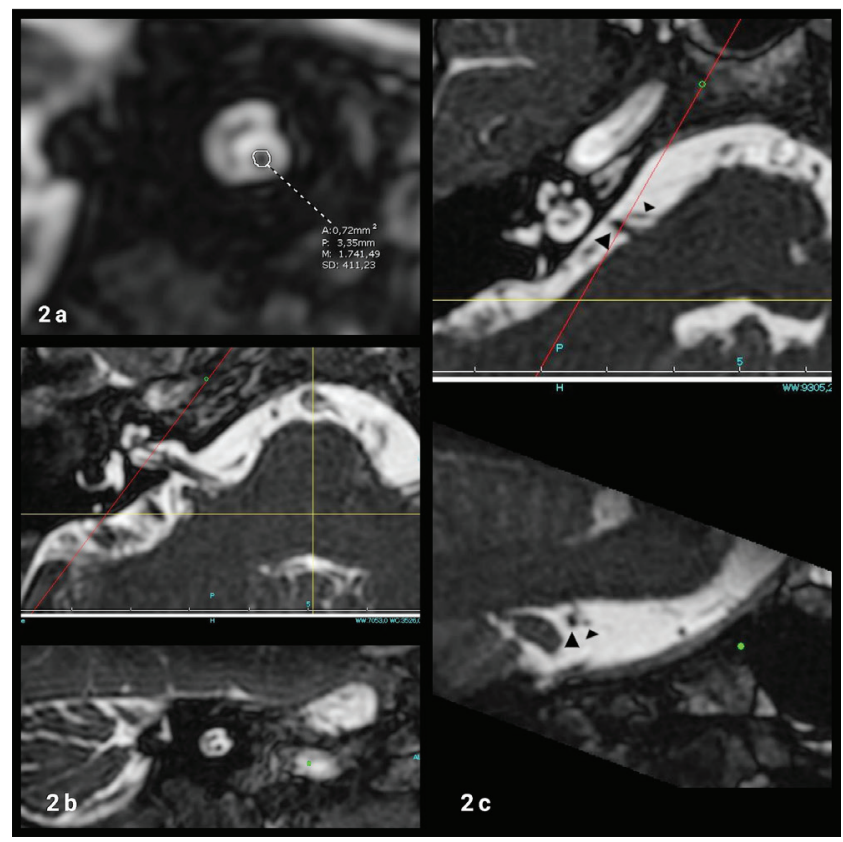

Figure 2. a. Cochlear nerve measurements in sagittal MPR FIESTA image (free-hand method) on MRI. b. Cochlear nerve in axial and sagittal MPR FIESTA image on MRI. c. Right vestibulocochlear complex (large arrowhead) and facial nerve (small arrowhead) on the axial and sagittal, oblique MPR images at the level of the brainstem on MRI

MRI: Magnetic resonance imaging, MPR: Multiplanar reconstruction 
standardeviation 12.6, a total of 42 ears) were included in the study.

The age and gender distribution of the patients in the CM group and the VM group did not differ significantly ( $p>0.05$ ) (Table 1).

\section{Computed Tomography Findings}

There was no statistically significant difference between $\mathrm{CM}$ and $\mathrm{VM}$ groups in terms of the right side of cochlear width; vestibular length and height; IAC proximal, mid-, and distal height; right IAC length; cochlear and vestibular aperture diameters $(p>0.05)$. Additionally, the distances between the right side of tegmen- mastoid tip, sigmoid sinus anterior wall - external acoustic meatus, and petrous apex-mastoid aditus medial wall did not differ significantly $(p>0.05)$ in the CM group and the VM group. However, right cochlear height in the VM group was significantly higher than the CM group $(p<0.05)$ (Table 2$)$.

There was no statistically significant difference between $\mathrm{CM}$ and VM groups in terms of the left side of cochlear width; vestibular length; IAC proximal, mid-, and distal height; right IAC length; cochlear and vestibular aperture diameters $(p>0.05)$. Additionally, the distances between the left side of tegmen-mastoid tip, sigmoid sinus anterior wall - external acoustic meatus, and petrous apex-mastoid aditus medial wall did not differ significantly $(p>0.05)$ in the CM group and the VM group. However, the left side of cochlear and vestibular heights in the VM group was significantly higher than the CM group $(p<0.05)$ (Table 2 ).

\section{Magnetic Resonance Imaging Findings}

The right side CSA of vestibulocochlear complex, facial nerve, superior vestibular nerve, inferior vestibular nerve, and cochlear nerve did not differ significantly $(p>0.05)$ in CM group and VM group (Table 3).

The left side CSA of vestibulocochlear complex, facial nerve, superior vestibular nerve, inferior vestibular nerve, and cochlear nerve did not differ significantly $(p>0.05)$ in CM group and VM group (Table 3).

\section{Discussion}

This research is the first study evaluating the anatomical structural differences of the inner ear and mastoid bone in $\mathrm{CT}$ examination between VM and CM headache, and the 7th and 8th nerves in MRI. In our study, no significant difference was found in CT measurements, between the VM group and CM group in terms of HRCT findings except for cochlear and vestibular heights. Statistically significant difference was found in cochlear and vestibular height in the VM group compared to the CM group. However, these differences were only in the right or left ears and not both. In MRI, no significant anatomical differences were found between the two groups.

Manzari L stated that VM and motion sickness was the most common accompanying diagnosis in patients who were found to have unilateral and bilateral large vestibular aqueduct on CT imaging (21).

Since the large vestibular aqueduct is a finding that can also be seen in Meniere's disease, some studies have been conducted to determine possible radiological differences between VM and Meniere's disease, and endolymphatic hydrops has been tried to be shown $(7,22,23)$.

Sun et al. (22) evaluated the usability of MRI for the investigation of endolymphatic hydrops in patients with definite or possible VM and Meniere's disease. Although varying degrees of cochlear and vestibular hydrops were observed in the affected ears of patients with Meniere's disease, suspicious cochlear hydrops was observed in patients with VM however vestibular hydrops was not found (22).

Nakadaka et al. (23) compared to the size of the cochlear and vestibular endolymphatic space between VM and Meniere's disease patients and no vestibular hydrops was observed except in 2 patients in the VM group. However significant vestibular hydrops was observed in all patients with Meniere's disease (23).

As an interesting result, Gurkov et al. (7) found cochlear and vestibular endolymphatic hydrops in some VM patients. It has been stated that locally developed inner ear MRI can be useful in the diagnosis of patients with suspected VM (7).

Due to the detection of the large vestibular aqueduct and possible hydrops in the radiological examinations of patients with VM, some studies have been conducted to investigate the difference between VM and Meniere's disease $(5,22,23)$. Krombach $\mathrm{G}$ et al. (24) investigated anatomical differences by $\mathrm{CT}$ in Meniere's patients. They found that the vestibular aqueduct was shorter and narrower and the left cochlear

\begin{tabular}{|c|c|c|c|c|c|c|c|c|c|c|c|}
\hline & & \multicolumn{4}{|c|}{ Chronic migraine } & \multicolumn{4}{|c|}{ Vestibular migraine } & \multirow{2}{*}{\multicolumn{2}{|c|}{$p$}} \\
\hline & \multicolumn{3}{|l|}{ Mean \pm SD n-\% } & \multirow{2}{*}{$\begin{array}{l}\text { Medyan } \\
12.6 \\
\end{array}$} & \multicolumn{3}{|c|}{ Mean \pm SD n-\% } & \multirow{2}{*}{\begin{tabular}{|l|} 
Medyan \\
12.8 \\
\end{tabular}} & \multirow[b]{2}{*}{44.0} & & \\
\hline \multicolumn{2}{|l|}{ Age } & 40.7 & \pm & & 45.0 & 40.7 & \pm & & & 0,962 & $\mathrm{~m}$ \\
\hline \multirow{2}{*}{ Gender } & Female & 18 & & $85.7 \%$ & & 15 & & $71.4 \%$ & & \multirow{2}{*}{0,117} & \multirow{2}{*}{$x^{2}$} \\
\hline & Male & 3 & & $14.3 \%$ & & 8 & & $38.1 \%$ & & & \\
\hline
\end{tabular}


height was significantly higher in Meniere's patients compared to the control group (24). Similarly, a significant difference was found in cochlear height and vestibular height in the VM group compared to the CM group in our study. The importance of morphological observations can be predicted after the final clarification of VM pathogenesis; however, anatomical observations can provide valuable clues regarding the pathogenesis of the disease. It is not yet clear that the VM is a physiopathologically and anatomically completely different entity from Meniere's disease. When viewed from this aspect, we observed in our study that there were anatomical differences in the inner ear similar to Meniere's patients.
The number of studies investigating the differences between $C M$ and $V M$ is limited in the literature $(10,25$ 28). Besides that, possible anatomical differences in the inner ear and mastoid antrum of patients with VM and $C M$ and in the IAC and the nerves within it have not been investigated before.

MRI can also help us to understand the morphological differences and pathogenesis between $\mathrm{VM}$ and $\mathrm{CM}$ headaches. In previous studies, hypermetabolism was detected in functional imaging of the brain, during and between migraine attacks. In functional MRI, signal changes due to activity involvement were encountered

\begin{tabular}{|c|c|c|c|c|c|c|c|c|c|c|}
\hline & \multicolumn{4}{|c|}{ Chronic migraine } & \multicolumn{4}{|c|}{ Vestibular migraine } & \multirow{2}{*}{\multicolumn{2}{|c|}{ p }} \\
\hline & \multicolumn{3}{|c|}{ Median \pm SD } & Median & \multicolumn{3}{|c|}{ Mean \pm SD } & Medyan & & \\
\hline \multicolumn{11}{|l|}{ Left (CT) } \\
\hline Cochlear width & 9.20 & \pm & 0.66 & 9.29 & 8.98 & \pm & 0.57 & 9.03 & 0.318 & m \\
\hline Cochlear height & 3.41 & \pm & 0.35 & 3.37 & 3.64 & \pm & 0.40 & 3.65 & 0.040 & $\mathrm{~m}$ \\
\hline Vestibular length & 5.66 & \pm & 0.48 & 5.66 & 5.68 & \pm & 0.39 & 5.60 & 0.906 & $\mathrm{~m}$ \\
\hline Vestibular height & 2.96 & \pm & 0.51 & 3.10 & 3.20 & \pm & 0.39 & 3.20 & 0.124 & $\mathrm{~m}$ \\
\hline IAC proximal height & 4.31 & \pm & 0.80 & 4.20 & 4.49 & \pm & 0.99 & 4.52 & 0.549 & $\mathrm{~m}$ \\
\hline IAC mid height & 4.68 & \pm & 0.81 & 4.77 & 4.78 & \pm & 0.93 & 4.51 & 0.897 & $\mathrm{~m}$ \\
\hline IAC distal height & 4.49 & \pm & 0.59 & 4.41 & 4.67 & \pm & 0.79 & 4.85 & 0.391 & m \\
\hline IAC length & 10.9 & \pm & 2.0 & 10.9 & 10.3 & \pm & 2.1 & 9.9 & 0.188 & m \\
\hline Cochlear apertura diameter & 2.19 & \pm & 0.29 & 2.20 & 2.12 & \pm & 0.38 & 2.15 & 0.716 & m \\
\hline Vestibular apertura diameter & 0.92 & \pm & 0.24 & 0.87 & 0.90 & \pm & 0.29 & 0.86 & 0.751 & m \\
\hline Distance between tegmen-mastoid tip & 36.5 & \pm & 3.8 & 36.5 & 36.2 & \pm & 4.6 & 36.0 & 1.000 & m \\
\hline $\begin{array}{l}\text { Distance between sigmoid sinus anterior wall - } \\
\text { external acoustic meatus }\end{array}$ & 12.4 & \pm & 4.5 & 13.0 & 11.7 & \pm & 2.7 & 11.2 & 0.206 & m \\
\hline $\begin{array}{l}\text { Distance between petrous apex-mastoid aditus } \\
\text { medial wall }\end{array}$ & 29.4 & \pm & 13.1 & 34.9 & 35.0 & \pm & 2.3 & 35.1 & 0.664 & m \\
\hline \multicolumn{11}{|l|}{ Right (CT) } \\
\hline Cochlear width & 9.10 & \pm & 0.56 & 9.21 & 9.06 & \pm & 0.48 & 9.01 & 0.716 & $\mathrm{~m}$ \\
\hline Cochlear height & 3.45 & \pm & 0.32 & 3.41 & 3.73 & \pm & 0.29 & 3.78 & 0.002 & $\mathrm{~m}$ \\
\hline Vestibular length & 5.58 & \pm & 0.39 & 5.50 & 5.63 & \pm & 0.42 & 5.65 & 0.526 & $\mathrm{~m}$ \\
\hline Vestibular height & 3.09 & \pm & 0.37 & 3.16 & 3.39 & \pm & 0.27 & 3.44 & 0.014 & $\mathrm{~m}$ \\
\hline IAC proximal height & 4.05 & \pm & 0.81 & 3.90 & 4.24 & \pm & 1.01 & 4.18 & 0.638 & $\mathrm{~m}$ \\
\hline IAC mid height & 4.68 & \pm & 0.92 & 4.48 & 4.77 & \pm & 1.16 & 4.40 & 0.934 & $\mathrm{~m}$ \\
\hline IAC distal height & 4.60 & \pm & 0.67 & 4.52 & 4.59 & \pm & 0.67 & 4.61 & 0.991 & $\mathrm{~m}$ \\
\hline IAC length & 10.4 & \pm & 2.1 & 10.5 & 9.9 & \pm & 2.0 & 9.9 & 0.250 & $\mathrm{~m}$ \\
\hline Cochlear apertura diameter & 2.05 & \pm & 0.29 & 2.11 & 2.13 & \pm & 0.38 & 2.15 & 0.581 & $\mathrm{~m}$ \\
\hline Vestibular apertura diameter & 0.91 & \pm & 0.31 & 0.77 & 0.98 & \pm & 0.26 & 0.86 & 0.209 & m \\
\hline Distance between tegmen-mastoid tip & 35.6 & \pm & 3.9 & 35.6 & 37.2 & \pm & 4.9 & 38.1 & 0.189 & m \\
\hline $\begin{array}{l}\text { Distance between sigmoid sinus anterior wall - } \\
\text { external acoustic meatus }\end{array}$ & 12.9 & \pm & 2.6 & 13.0 & 12.5 & \pm & 2.6 & 12.3 & 0.557 & m \\
\hline $\begin{array}{l}\text { Distance between petrous apex- mastoid aditus } \\
\text { medial wall }\end{array}$ & 36.3 & \pm & 2.4 & 36.4 & 36.0 & \pm & 5.0 & 35.3 & 0.177 & m \\
\hline
\end{tabular}


in the thalamus and occipital lobes of patients with VM $(10,25)$.

Wang et al. (26) investigated the differences in cerebral gray matter in CM patients without aura, VM and healthy controls. The gray matter volume of some brain regions of patients with VM was found to be significantly greater than the other two groups (26).

Zhe et al. (28) compared the healthy control group and VM group and examined structural cortical changes with MRI. Interestingly, they found that the volume of gray matter decreased in the different brain regions in the VM group compared to the control group (28).

In our study, no significant anatomical structure differences were found in the MRI of 7th and 8th nerves between both groups.

In a CT study, it was shown that the morphometric anatomy of the semicircular canals was compatible with the movement pattern. It has been stated that the diameter of the semicircular canals is more important than the radius of curvature and the height, width and length of the semicircular canals (29).

It has been shown that there is a high level of morphological variation in the bony labyrinth of the inner ear, especially in the shape, size, and angles of the semicircular canals, between very slow-moving sloth species and much faster moving species (30).

Are the different movement patterns in different species a consequence of this anatomical structural difference or have the inner ear anatomies adapted to the modality of movement phylogenetically over time because they act differently? Unlike healthy individuals, do minimal anatomical differences lead to significant balance and movement disorders in Meniere's disease, VM and other vertiginous diseases? All these issues need to be illuminated with further comprehensive studies.

\section{Study Limitations}

There were 13 structural comparisons and right and left ears were compared separately but some differences were only in the right or left ears and not both, which is the most weak part of the present study. We thought that the reason might be the small sample. Therefore, the small number of patients and radiological and clinical long-term follow-up failure are the limitations of this study. There is a need for more comprehensive studies to be conducted with a much larger number of patients on whether minor anatomical structural differences cause major functional debility and taking the early and long-term follow-up results into account.

On the other hand, another limitation of this study is that an audiometric evaluation was not performed to consider the presence of low frequency sensorineural hearing loss, which helps in the differential diagnosis of Meniere's disease in the patient group diagnosed with vestibular migraine. However, it should not be neglected that permanent hearing loss in Menier's disease occurs after repeated attacks over time. Hearing status should be evaluated in patients with longterm follow-up to investigate the possibility of VM being a variant of Menier's disease. The follow-up period in this study was limited to 3 years and this is one of the limitations of our study. We need to evaluate the long-term hearing status in patients with VM in future studies.

\section{Conclusion}

A significant difference was found in cochlear and vestibular heights of the right or left ears in the VM group

\begin{tabular}{|c|c|c|c|c|c|c|c|c|c|c|}
\hline & \multicolumn{4}{|c|}{ Chronic migraine } & \multicolumn{4}{|c|}{ Vestibular migraine } & \multirow{2}{*}{\multicolumn{2}{|c|}{$\mathbf{p}$}} \\
\hline & \multicolumn{3}{|c|}{ Mean \pm SD } & Median & \multicolumn{3}{|c|}{ Mean \pm SD } & Median & & \\
\hline \multicolumn{11}{|l|}{ Left (MRI) } \\
\hline Vestibulocochlear complex CSA & 2.83 & \pm & 0.65 & 2.76 & 2.86 & \pm & 0.42 & 2.81 & 0.549 & $\mathrm{~m}$ \\
\hline Facial nerve CSA (CPA) & 0.97 & \pm & 0.33 & 0.93 & 1.19 & \pm & 0.41 & 1.09 & 0.076 & $\mathrm{~m}$ \\
\hline Superior vestibular nerve & 0.64 & \pm & 0.20 & 0.60 & 0.66 & \pm & 0.23 & 0.65 & 0.897 & $\mathrm{~m}$ \\
\hline Inferior vestibular nerve & 0.41 & \pm & 0.16 & 0.33 & 0.43 & \pm & 0.15 & 0.43 & 0.533 & $\mathrm{~m}$ \\
\hline IAC cochlear nerve & 1.07 & \pm & 0.31 & 0.96 & 1.02 & \pm & 0.41 & 0.88 & 0.301 & $\mathrm{~m}$ \\
\hline \multicolumn{11}{|l|}{ Right (MRI) } \\
\hline Vestibulocochlear complex CSA & 2.93 & \pm & 0.57 & 2.85 & 2.85 & \pm & 0.56 & 2.81 & 0.760 & $\mathrm{~m}$ \\
\hline Facial nerve CSA (CPA) & 0.87 & \pm & 0.31 & 0.73 & 0.96 & \pm & 0.22 & 0.95 & 0.076 & $\mathrm{~m}$ \\
\hline Superior vestibular nerve & 0.67 & \pm & 0.23 & 0.63 & 0.65 & \pm & 0.26 & 0.59 & 0.672 & $\mathrm{~m}$ \\
\hline Inferior vestibular nerve & 0.38 & \pm & 0.12 & 0.35 & 0.43 & \pm & 0.19 & 0.38 & 0.580 & $\mathrm{~m}$ \\
\hline IAC cochlear nerve & 1.00 & \pm & 0.25 & 0.89 & 0.93 & \pm & 0.24 & 0.95 & 0.341 & $\mathrm{~m}$ \\
\hline
\end{tabular}


compared to the CM group. There may be a relationship between the morphological changes caused by anatomical structural differences between CM and VM and functional impairment. As the differences were not found in both ears, future studies with larger samples should be performed.

\section{Author Contributions}

Concept: Z.C., C.K.E., O.Y., Y.K., O.O.Y., Design: Z.C., C.K.E., O.Y., Y.K., O.O.Y., Data Collection or Processing: Z.C., C.K.E., O.Y., Y.K., O.O.Y., Analysis or Interpretation: Z.C., C.K.E., O.Y., Y.K., O.O.Y., Literature Search: Z.C., C.K.E., O.Y., Y.K., O.O.Y., Writing: Z.C., C.K.E., O.Y., Y.K., O.O.Y.

Funding and support:This research received no specific grant from any funding agency in public, commercial or not-for-profit sectors.

Conflict of interests: The authors declare that there is no conflict of interest.

\section{References}

1. Headache Classification Committee of the International Headache Society (IHS) The International Classification of Headache Disorders, 3rd edition. Cephalalgia 2018;38:1-211.

2. Yeh WZ, Blizzard L, Taylor BV. What is the actual prevalence of migraine? Brain Behav 2018;8:950.

3. Burch RC, Buse DC, Lipton RB. Migraine: Epidemiology, Burden, and Comorbidity. Neurol Clin 2019;37:631-49.

4. Dieterich $\mathrm{M}$, Obermann $\mathrm{M}$, Celebisoy $\mathrm{N}$. Vestibular migraine: the most frequent entity of episodic vertigo. I Neurol 2016;263:82-9.

5. Bisdorff AR. Management of vestibular migraine. Ther Adv Neurol Disord 2011;4:183-91.

6. Swaminathan A, Smith JH. Migraine and vertigo. Curr Neurol Neurosci Rep 2015;15:515.

7. Gürkov R, Kantner C, Strupp M, Flatz W, Krause E, ErtlWagner B. Endolymphatic hydrops in patients with vestibular migraine and auditory symptoms. Eur Arch Otorhinolaryngol 2014;271:2661-7.

8. Kirsch V, Becker-Bense S, Berman A, Kierig E, Ertl-Wagner B, Dieterich M. Transient endolymphatic hydrops after an attack of vestibular migraine: a longitudinal single case study. J Neurol 2018;265:51-3.

9. Carvalho GF, Vianna-Bell FH, Florencio LL, et al. Presence of vestibular symptoms and related disability in migraine with and without aura and chronic migraine. Cephalalgia 2019;39:29-37.

10. Russo A, Marcelli V, Esposito $F$, et al. Abnormal thalamic function in patients with vestibular migraine. Neurology 2014;82:2120-6.
11. Obermann M, Wurthmann S, Steinberg BS, Theysohn N, Diener HC, Naegel S. Central vestibular system modulation in vestibular migraine. Cephalalgia 2014;34:1053-61.

12. Yalin OÖ, Uludüz D, Sungur MA, Sart H, Özge A. Identification of Allodynic Migraine Patients with the Turkish Version of the Allodynia Symptom Checklist: Reliability and Consistency Study. Noro Psikiyatr Ars 2017;54:260-6.

13. Marcus S, Whitlow CT, Koonce J, et al. Computed tomography supports histopathologic evidence of vestibulocochlear sexual dimorphism. Int J Pediatr Otorhinolaryngol 2013;77:1118-22.

14. Purcell DD, Fischbein N, Lalwani AK. Identification of previously "undetectable" abnormalities of the bony labyrinth with computed tomography measurement. Laryngoscope 2003;113:1908-11.

15. Purcell D, Johnson J, Fischbein N, Lalwani AK. Establishment of normative cochlear and vestibular measurements to aid in the diagnosis of inner ear malformations. Otolaryngol Head Neck Surg 2003;128:78-87.

16. Lan MY, Shiao JY, Ho CY, Hung HC. Measurements of normal inner ear on computed tomography in children with congenital sensorineural hearing loss. Eur Arch Otorhinolaryngol 2009;266:1361-4.

17. Teissier N, Van Den Abbeele T, Sebag G, Elmaleh-Berges M. Computed Tomography measurements of the normal and the pathologic cochlea in children. Pediatr Radiol 2010;40:27583.

18. Fatterpekar GM, Mukherji SK, Alley J, Lin Y, Castillo M. Hypoplasia of the bony canal for the cochlear nerve in patients with congenital sensorineural hearing loss: initial observations. Radiology 2000;215:243-6.

19. McClay JE, Tandy R, Grundfast K, et al. Major and minor temporal bone abnormalities in children with and without congenital sensorineural hearing loss. Arch Otolaryngol Head Neck Surg 2002;128:664-71.

20. Flatz WH, Henneberger A, Reiser MF, Gürkov R, Ertl-Wagner B. In Vivo Morphometric Analysis of Human Cranial Nerves Using Magnetic Resonance Imaging in Menière's Disease Ears and Normal Hearing Ears. J Vis Exp 2018;:57091.

21. Manzari L. Vestibular signs and symptoms of volumetric abnormalities of the vestibular aqueduct. J Laryngol Otol 2008;122:557-63.

22. Sun W, Guo P, Ren T, Wang W. Magnetic resonance imaging of intratympanic gadolinium helps differentiate vestibular migraine from Ménière disease. Laryngoscope 2017; 127:2382-8.

23. Nakada T, Yoshida T, Suga K, et al. Endolymphatic space size in patients with vestibular migraine and Ménière's disease. J Neurol 2014;261:2079-84.

24. Krombach GA, van den Boom M, Di Martino E, et al. Computed tomography of the inner ear: size of anatomical structures in the normal temporal bone and in the temporal bone of patients with Menière's disease. Eur Radiol 2005;15:1505-13. 
25. ElSherif M, Reda MI, Saadallah H, Mourad M. Eye movements and imaging in vestibular migraine. Acta Otorrinolaringol Esp 2020;71:3-8.

26. Wang $S$, Wang $H$, Zhao $D$, et al. Grey matter changes in patients with vestibular migraine. Clin Radiol 2019;74:898.

27. Messina R, Rocca MA, Colombo B, et al. Structural brain abnormalities in patients with vestibular migraine. J Neurol 2017;264:295-303.

28. Zhe X, Gao J, Chen L, et al. Altered structure of the vestibular cortex in patients with vestibular migraine. Brain Behav 2020;10:1572
29. Pfaff C, Czerny S, Nagel D, Kriwet J. Functional morphological adaptations of the bony labyrinth in marsupials (Mammalia, Theria). J Morphol 2017;278:742-9.

30. Billet G, Hautier L, Asher RJ, et al. High morphological variation of vestibular system accompanies slow and infrequent locomotion in three-toed sloths. Proc Biol Sci 2012;279:39329. 